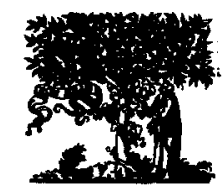

ELSEVIER

BioSysterns 37 (1996) 3-17

\title{
Spatialization and greater generosity in the stochastic Prisoner's Dilemma
}

\author{
Patrick Grim \\ Group for Logic and Formal Semantics, Department of Philosophy, SUNY at Stony Brook, Stony Brook, New York, \\ NY 11794, USA
}

\begin{abstract}
The iterated Prisoner's Dilemma has become the standard model for the evolution of cooperative behavior within a community of egoistic agents, frequently cited for implications in both sociology and biology. Due primarily to the work of Axelrod (1980a, 1980b, 1984, 1985), a strategy of tit for tat (TFT) has established a reputation as being particularly robust. Nowak and Sigmund (1992) have shown, however, that in a world of stochastic error or imperfect communication, it is not TFT that finally triumphs in an ecological model based on population percentages (Axelrod and Hamilton 1981), but 'generous tit for tat' (GTFT), which repays cooperation with a probability of cooperation approaching 1 but forgives defection with a probability of $1 / 3$. In this paper, we consider a spatialized instantiation of the stochastic Prisoner's Dilemma, using two-dimensional cellular automata (Wolfram, 1984, 1986; Gutowitz, 1990) to model the spatial dynamics of populations of competing strategies. The surprising result is that in the spatial model it is not GTFT but still more generous strategies that are favored. The optimal strategy within this spatial ecology appears to be a form of 'bending over backwards', which returns cooperation for defection with a probability of $2 / 3$ - a rate twice as generous as GTFT.
\end{abstract}

Keywords: Prisoner's Dilemma; Game theory; Altruism; Generosity

\section{The classical work}

In the classical Prisoner's Dilemma, each of two players has an option to cooperate or defect. The traditional payoff matrix is Axelrod's, giving a reward $R$ of 3 points to each player for mutual cooperation, a penalty payoff $P$ of 1 for mutual defection, a temptation $T$ of 5 points for a player who defects while his opponent cooperates, and the sucker's payoff $S$ of 0 points to a player who cooperates while his opponent defects. In the iterated Prisoner's Dilemma, the process is repeated, and one can characterize general strategies for continued play: AllD, which defects regardless of the other's play, AllC, which cooperates regardless, 'grim' strategies which revert to constant defection once crossed, and tit for tat (TFT), which begins by cooperating, cooperates following a cooperation on the other side, and defects following a defection. 'One-deep' or 'reac- 
tive' strategies, which depend only on the opponent's play in the previous round, can be characterized by triples $\langle i, p, q\rangle$ where $i$ indicates the starting play, and $p$ and $q$ are the probabilities of cooperative response to cooperation and defection respectively. AllD can thus be represented by $\langle 0,0,0\rangle$, AllC by $\langle 1,1,1\rangle$, and TFT by $\langle 1,1,0\rangle$. In the alternative hierarchical rule representation of Crowley (1995, this issue), AllD appears as the single rule /:D, AllC as /:C, and TFT as:

$/: \mathrm{C}$

/:D:D.

TFT was the clear victor in both of Axelrod's classic round-robin tournaments of submitted strategies (Axelrod, 1980a, 1980b, 1984, 1985). TFT also displaced all competing rules in an ecological model in which strategies 'reproduce' on any round as a function of their success against other strategies weighted by proportional representation on the previous round (Axelrod and Hamilton, 1981). TFT is collectively stable in the sense that no single mutation can invade it by strategic advantage. It isn't alone in this regard, of course: AllD is collectively stable as well ${ }^{1}$.

Though single mutations of TFT cannot invade AllD, Axelrod showed that a 'cluster' of TFT can, where 'cluster' is defined in terms of a higher probability of interaction between members of the cluster (Axelrod and Hamilton, 1981; Axelrod, 1984). As noted below, this last fact offers an intriguing comparison with some aspects of the present results.

The classic Axelrod results clearly award general high marks to TFT. More recent work, however, suggests that within more realistic constraints it is not TFT but another family of strategies that should be regarded as the ultimate winners.

\section{The Prisoner's Dilemma in an imperfect world}

Nowak and Sigmund (1992) concentrate on one-deep stochastic strategies, confined to consideration of a single previous move by an opponent but which assign probabilities for coopera- tion or defection. Different strategies can thus be envisaged in terms of ordered triples $\langle i, p, q\rangle$ with $i$ as the probability of cooperation in the initial round, $p$ as the probability of cooperation following a cooperative move by an opponent, and $q$ as the probability of cooperation following an opponent's defection. Classical TFT would become $\langle 1,1,0\rangle$ as before, AllD would become $\langle 0,0,0\rangle$. But we might also introduce other possi-

\footnotetext{
${ }^{1}$ Despite some confusion in the literature, however, neither TFT nor ALLD strictly qualifies as an 'evolutionarily stable strategy' or ESS in the sense of Maynard Smith $(1982,1986)$. Representing the payoff for a player $x$ against a player $y$ in a given series of games as $\mathrm{P}(x, y)$, Maynard Smith's formal definition specifies that a strategy $x$ is evolutionarily stable just in case $\mathrm{P}(x, x)>\mathrm{P}(y, x)$ or both $\mathrm{P}(x, x)=\mathrm{P}(y, x)$ and $\mathrm{P}(x, y)>\mathrm{P}(y, y)$ for all strategies $y \neq x$. It is true that both TFT and AlID resist invasion in that no other strategy can get a higher score against TFT or AllD than they get against themselves and at the same time get an equal or higher scorc against itself than they get against it. But there are strategies which do precisely as well against TFT and AllD as they do against themselves and which also do as well with their own kind as TFT and AllD do with them: AllC does as well in a context of TFT as TFT does, for example, and 'Suspicious Tit For Tat' (STFT) does as well in a context of AllD as AllD does. The conditions of Maynard Smith's formal definition are thus not fulfilled for either TFT or AllD, and neither qualifies as an ESS. With an eye to natural selection, as Nowak and Sigmund note, although such strategies cannot invade by strategic advantage, they can invade by something like genetic drift (Nowak and Sigmund 1989a, 192; 1989b, 22).

Axelrod (1984) labels a strategy $x$ 'collectively stable' just in case there is no alternative strategy $y$ such that $\mathrm{P}(y, x)>$ $\mathrm{P}(x, x)$, and notes that this is equivalent to $x$ being in Nash equilibrium with itself. Nowak (1990) labels a strategy $x$ 'incapable of invasion by selection pressure' if $\mathrm{P}(x, x)>$ $\mathrm{P}(y, x)$ or $\mathrm{P}(x, x)=\mathrm{P}(y, x)$ and $\mathrm{P}(x, y) \geq \mathrm{P}(y, y)$ for all alternative strategies $y$. Although they do not qualify as ESSs, it is clear that TFT and AllD are in these senses buth collectively stable and incapable of invasion by selection pressure.

As all this should make clear, notions of stability against invasion call for careful handling. For further discussion see esp. Nowak (1990a) and a particularly clear exposition in Skyrms (1992). As indicated later in the paper, notions of stability also need to be handled carefully in extension to fully spatial models. It should finally be noted that stable strategies in any of the senses above must be distinguished from 'attractor' strategies, toward which a system may be drawn. As indicated in Nowak (1990b), these are in principle distinct.
} 
bilities, including various degrees of generous TFT (GTFT) such as $\langle 1,1,0.1\rangle$ and $\langle 1,1,1 / 3\rangle$, each of which begins with cooperation and rewards cooperation with full cooperation, but each of which is generous in forgiving defection with a probability of 0.1 and $1 / 3$ respectively. In terms of hierarchical rules (Crowley 1995, this issue), GTFT with a forgiveness rate of $1 / 3$ takes the following form:

$/: \mathrm{C}$

$/ \mathrm{D}: \mathrm{D}$

$/ \mathrm{D}: \mathrm{D}$

$/ \mathrm{D}: \mathrm{C}$

With an assumption of infinite games between contenders, Nowak and Sigmund are able to simplify further by ignoring the role of the initial play $i^{2}$.

Using Axelrod and Hamilton's (1981) technique of updating strategy proportions in a popu-

\footnotetext{
${ }^{2}$ Crowley, Provencher, and Sloane el al. (1995, this issue) seem to express reservations about infinitely iterated games, but in many contexts - including the present - infinite games can be seen merely as a convenient mathematical fiction. In an iterated game between two deterministic strategies of simple sorts, for example, a periodic pattern of play will inevitably be established. The value for an 'infinite' game for one of these players is simply the average gain per play across that repeated period, and thus represents a value which games of increasing finite length will approach. Although the play of stochastic strategies of the sort at issue cannot be expected to be periodic, scores for an 'infinite' game here too represent merely the limits for which games of increasing length will approach. In both the deterministic and stochastic case, on the other hand, any choice for a finite limit is bound to face dangers of arbitrariness. In the deterministic case, these include: (a) the inevitable arbitrariness of how much an initial segment before periodicity is established will count; (b) (for sufficiently complex assortments of strategies, at least) the arbitrariness of different cuts for periods of different lengths, counting perhaps only the first play of a final period, with scores 055 for a certain player because the finite limit happens to fall immediately after the 0 . In the present case, for simple stochastic strategies, any finite limit would give arbitrary answers to: (a) the importance accorded to deterministic first moves; (b) how probabilities happen to be expressed within the sample run. The mathematical fiction of an 'infinite game' is a convenient way of avoiding both of these difficulties, both in Nowak and Sigmund's work and in the results below.
}

lation on the basis of relative success in the previous round, Nowak and Sigmund report an evolution in which TFT plays a crucial pivotal role but in which it is GTFT rather than TFT that proves the ultimate winner.

With $n=100$ different reactive strategies uniformly distributed on the unit square, evolution proceeds in most cases towards AllD: those $(p, q)$-strategies from the sample which are closest to $(0,0)$ increase in frequency, while all others vanish. ... The outcome alters dramatically if one of the initial strategies (added by hand or by chance), is TFT, or very close to it ... The first phase is practically indistinguishable from the previous run. The strategies near AllD grow rapidly. TFT and all other reciprocating strategies (near $(1,0)$ ) seem to have disappeared. But an embattled minority remains and fights back. The tide turns when 'suckers' are so decimated that exploiters can no longer feed on them. Slowly at first, but gathering momentum, the reciprocators come back, and the exploiters now wane. But the TFT-like strategy that caused this reversal of fortune is not going to profit from it: having eliminated the exploiters, it is robbed of its mission and superseded by the strategy closest to GTFT (with $\langle p, q\rangle=$ $\langle 1,1 / 3\rangle$ ). Evolution then stops (Nowak and Sigmund 1992 , 252).

Their general characterization is as follows:

We find that a small fraction of TFT players is essential for the emergence of reciprocation in a heterogeneous population, but only paves the way for a more generous strategy. TFT is the pivot, rather than the aim, of an evolution towards cooperation. (250)

Here it is important to stress, however, that Nowak and Sigmund's is a pool of strategies envisaged as interacting in a world of inevitable error and imperfect communication. For that reason, none of the triplets $\langle i, p, q\rangle$ used involves a full probability of 0 or 1 in any position: references to TFT and AllD above, for example, must be read as references to their instantiation in an imperfect world, in which they appear only in the guise of stochastically imperfect variations such as $\langle 0.99,0.01\rangle$ and $\langle 0.01,0.01\rangle$. Nowak and Sigmund assume games between strategies of infinite length in which initial values can be ignored, calculated in terms of a payoff formula for strategies $s_{1}=\left\langle p_{1}, q_{1}\right\rangle$ and $s_{2}=\left\langle p_{2}, q_{2}\right\rangle$ as follows:

$\mathrm{V}\left(s_{1}\right.$ vs $\left.s_{2}\right)=1+4 c^{\prime}-c-c c^{\prime}$ 
where

$c=\left[q_{1}+\left(p_{1}-q_{1}\right) q_{2}\right] /\left[1-\left(p_{1}-q_{1}\right)\left(p_{2}-q_{2}\right)\right]$

$c^{\prime}=\left[q_{2}+\left(p_{2}-q_{2}\right) q_{1}\right] /\left[1-\left(p_{2}-q_{2}\right)\left(p_{1}-q_{1}\right)\right]$.

The assumption that initial values can be ignored, however, makes sense only if full values of $\mathbf{0}$ and 1 are disallowed in accord with the assumption of a world of imperfect information ${ }^{3}$. The payoff formula above is in fact mathematically undefined for crucial values of 0 and 1 .

What if a pure TFT, without communication error, were somehow included in Nowak and Sig-

\footnotetext{
${ }^{3}$ Consider, for example, strategies $\langle 0,1,0\rangle$ and $\langle 1,1,0\rangle$, which differ only in their initial value. Against itself, the first will achieve a constant score of 0 in an infinite game; against itself, the second will achieve a constant score of 1 .
}

mund's sample? A pure TFT would quite predictably block the evolution that Nowak and Sigmund trace towards more generous forms. No more generous strategy $\langle 1,1, X\rangle$ for $\mathrm{X}>0$ would grow strategically in an environment occupied by $\langle 1,1,0\rangle$ because payoffs for any such GTFT against TFT would be precisely the same as those for TFT against itself. Were a genuinely errorless TFT included in the sample, then it could be expected not only to take possession but to stubbornly maintain it.

Nowak and Sigmund's work should therefore not be read as in any way contradicting the classic Axelrod results. The world of Nowak and Sigmund's model is simply a different world from that of earlier models. It's a gritty world of ubiquitous and inevitable stochastic noise, and the failure for TFT reported for such a world is simply a failure of a stochastically imperfect in-
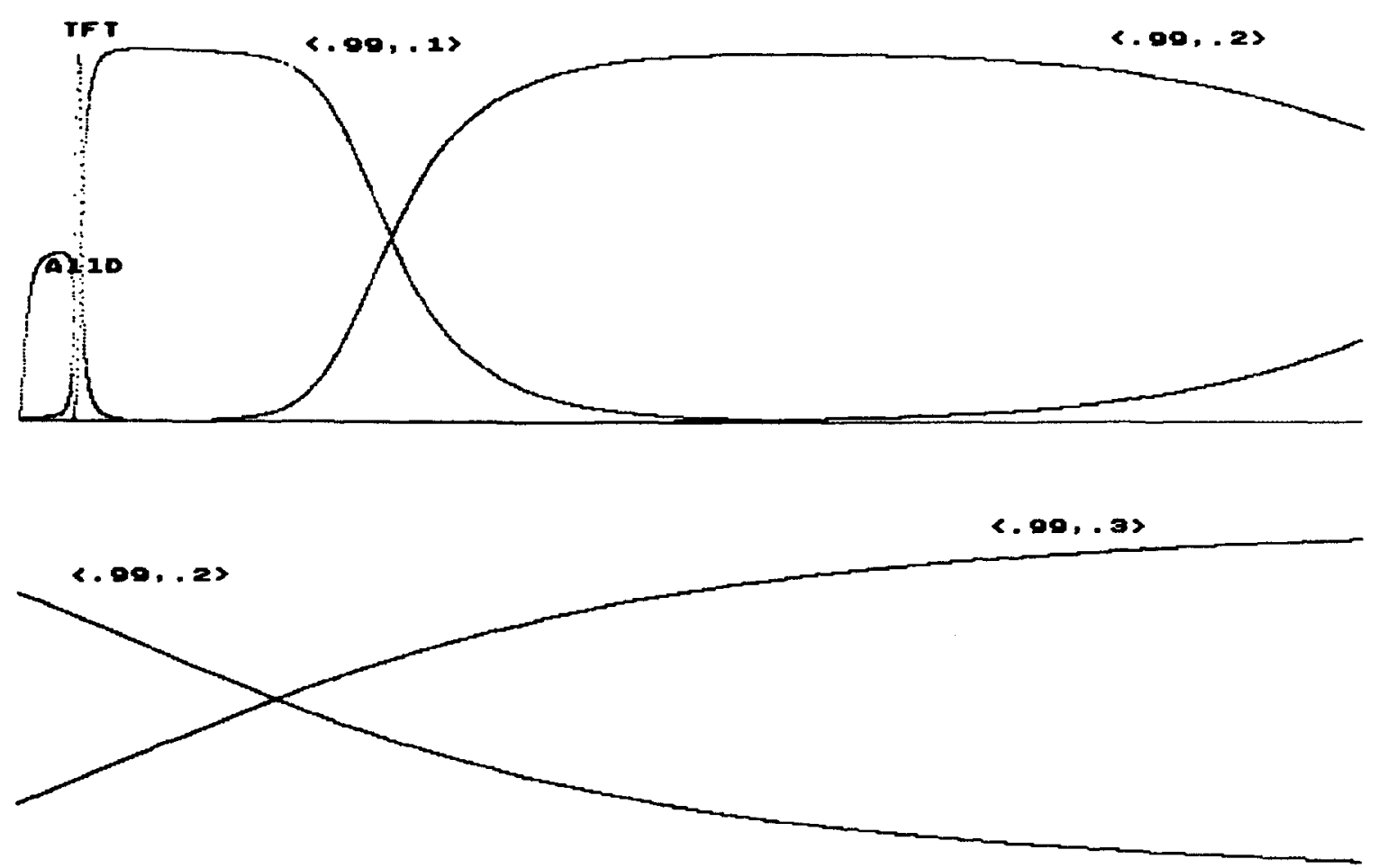

Fig. 1. The Nowak and Sigmund result: evolution toward GTFT in a world of imperfect information. Population proportions for labelled strategies shown over 12000 generations for an initial pool of 121 strategies $\langle p, q\rangle$ at 0.1 intervals, full values of 0 and 1 replaced with 0.01 and 0.99 . 
stantiation of the classic strategy. That failure alone should perhaps not be too surprising: as Molander (1985) showed, and Nowak and Sigmund (1992) note, the presence of any amount of stochastic noise is sufficient in the long run to reduce the payoff for two TFT players to precisely that of two random players.

In Fig. 1, we reproduce the Nowak and Sigmund result using a population of 121 purely stochastic strategies $\langle p, q\rangle$ at 0.1 intervals, with full values of 0 and 1 replaced with 0.01 and 0.99 , giving us a pool of strategies $\langle 0.01,0.01\rangle$, $\langle 0.01,0.1\rangle,\langle 0.01,0.2\rangle, \quad \ldots .\langle 0.99,0.9\rangle$, $\langle 0.99,0.99\rangle$. Each strategy plays all others represented in an infinitely iterated Prisoner's Dilemma in accordance with the payoff formula outlined above. At each generation $n+1$, the proportion $p_{n+1}(s)$ of a strategy, $s$, is computed as a function of its previous proportion $p_{n}(s)$ and its success against represented strategies, $m$, weighted by their proportions $p_{n}(m): p_{n+1}(s)=f_{n+1}(s) /$ $\Sigma f_{n+1}(m)$ for all strategies $\mathrm{m}$, where for any strategy $s f_{n+1}(s)=p_{n}(s) * \Sigma\left(V(s, m) * p_{n}(m)\right)$ for all strategies $m$.

Twelve thousand generations are shown. Stochastically imperfect AlID and its relatives are early winners, but arc effectively eliminated by stochastically imperfect TFT, $\langle 0.99,0.01\rangle$, by the 250 th generation. It is unable to sustain its victory, however, and is progressively supplanted by more generous strategies: $\langle 0.99,0.1\rangle,\langle 0.99,0.2\rangle$, and finally $\langle 0.99,0.3\rangle$. At this point, evolution stops. Most remarkable is the fact that evolution not only proceeds beyond stochastically imperfect TFT, but proceeds in such clear steps, with $\langle 0.99,0.1\rangle$ achieving clear dominance before $\langle 0.99,0.2\rangle$ even begins its rise ${ }^{4}$.

\section{The spatialized Prisoner's Dilemma}

An argument can clearly be made that the stochastic imperfection characteristic of Nowak and Sigmund's model is an aspect of realism: that

\footnotetext{
${ }^{4}$ Dugatkin and Mesterton-Gibbons (1995, this issue) suggest that egg-swapping fish may use a strategy similar to GTFT.
}

both biological and sociological worlds being modelled are gritty worlds of error and imperfect information, quite properly reflected in a model in which stochastic noise is unavoidable. The success of a pure TFT in a classical model, clinically free of error, though formally unchallenged, becomes less interesting from an applicational standpoint.

Here we want to add just one further aspect of realism to this model. Talk of clustering in previous work suggests a spatial model for the Prisoner's Dilemma, in which we envisage an array of players with different strategies interacting with their immediate neighbors ${ }^{5}$.

This is precisely the kind of model obtained if competing strategies are instantiated as a two-dimensional array of cellular automata (Wolfram 1984, 1986; Demongeot, Golés and Tchuente, 1985; Gutowitz, 1990; Mar and St. Denis, 1994). A spatial model of this sort applied merely to deterministic AllD and AllC appears in Nowak and May (1992, 1993); although initiated independently, the present work can be seen as a further application to questions of generosity in the richer stochastic ecology of Nowak and Sigmund (1992).

We envisage each cell of a two-dimensional array as playing against each of its neighbors and obtaining a local score. Each cell then surveys its neighbors. If none has a higher score, it retains its original strategy. If it has a neighbor or neighbors with higher scores, on the other hand, it converts to a neighboring strategy with the highest score, or is replaced by that strategy, depending on one's perspective. In the case of two neighbors with equal scores higher than that of the central cell, the strategy of one is chosen randomly. The resultant model is one in which success is in all cases computed against local competitors, with reproduction proceeding locally as well - both features, we think, which constitute a very simple

\footnotetext{
${ }^{5}$ Oliphant (1994) argues that some familiar characteristics of simpler forms of the Prisoner's Dilemma, including the superiority of defection in the non-iterated game, are contingent on the use of non-spatialized models. The small measure of realism afforded simply by spatialization can change things - a lesson emphasized here with respect to generosity for the stochastic case.
} 

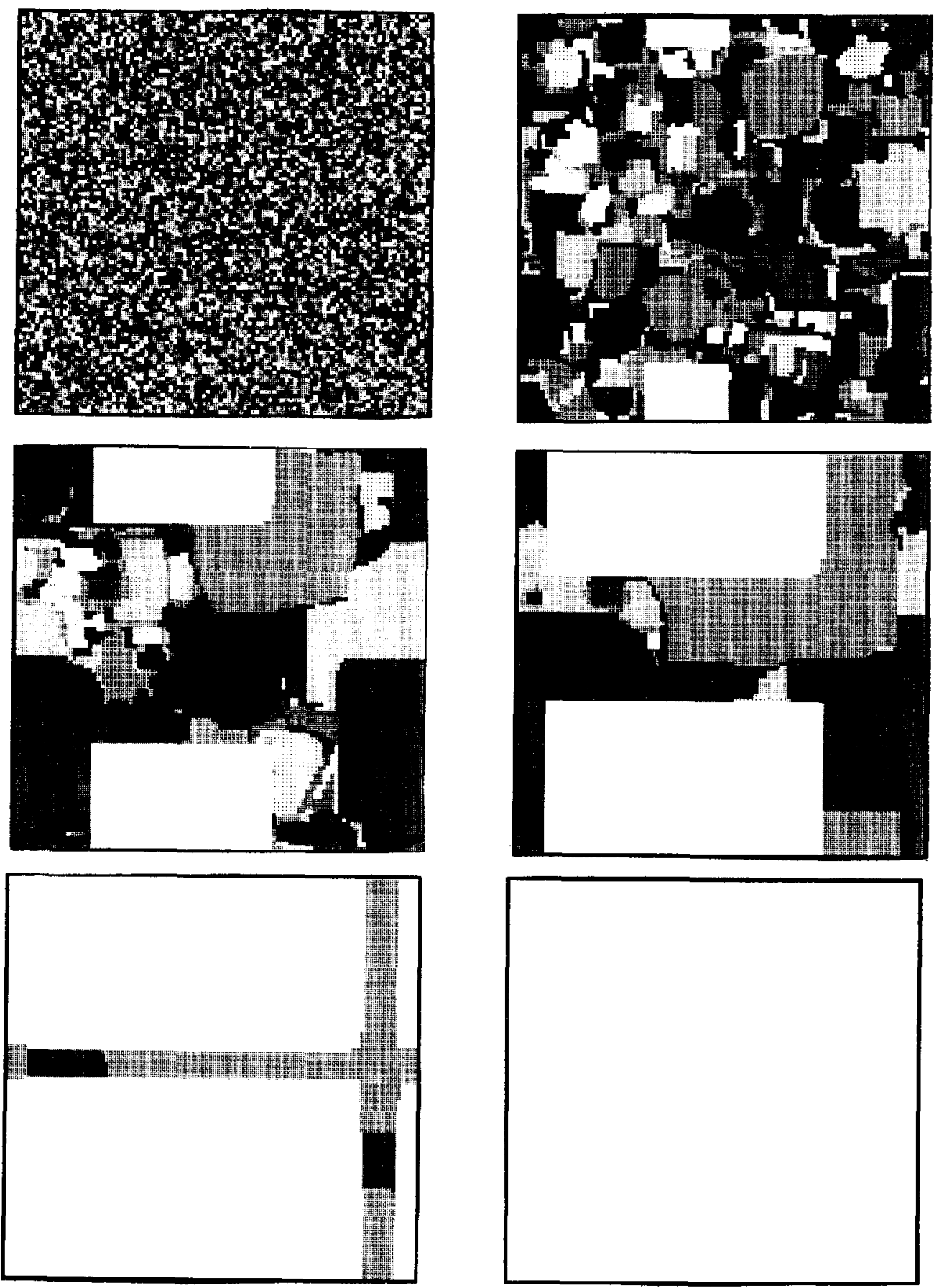

major players:

$\langle .99, .10\rangle$

$\langle .80, .20\rangle$

$\langle .90, .40\rangle$

$\langle .60, .20\rangle$

$\langle .50, .20\rangle$

Fig. 2. The spatialized Prisoner's Dilemma, showing a typical evolution from a randomized array of 121 stochastic strategies to conquest by $\langle 0.99,0.10\rangle$. Frames are at 12-generations intervals. 
measure of greater realism with an eye to either biological or sociological application.

Here it should be emphasized that virtually all we have done is to spatialize the Prisoner's Dilemma; in all other regards, we retain the stochastic model as it appears in Nowak and Sigmund ${ }^{6}$.

Part of the interest of the results regarding generosity below is the fact that they arise almost purely from this simple two-dimensional spatialization of an older paradigm, rather than from other intriguing but more complex variations explored elsewhere in this issue: individual recognition or non-play options (Crowley, Provencher, Sloane et al., 1995; Ashlock, Smucker, Stanley, and Tesfatsion, 1995; Orbell, Runde, and Morikawa 1995, this issue), epistemic modelling or reinforcement learning (Moon, Frost, and Stirling, 1995; Sandholm and Crites 1995, this issue), mutation by way of genetic algorithms or classifier systems (Crowley 1995; Crowley, Provencher, Sloane et al, 1995; Oliphant, 1995, this issue), or attempts to capture other aspects of natural evolution (Dugatkin and Mesterton-Gibbons, 1995, this issue). The importance of one-dimensional spatialization for the emergence of generosity in the context of merely deterministic strategies AllC and AllD is emphasized in Oliphant (1994); a similar importance for deterministic communication systems is emphasized in Oliphant (1995), (this issue). The work that follows reinforces Oliphant's general conclusions by showing an evolution of greater generosity in the two-dimensional spatialization of the stochastic Prisoner's Dilemma?.

In a first simple study, we used the same 121 strategies as in the Nowak and Sigmund replication above, once again playing each other in infinite games, but here randomly instantiated as cells of a $100 \times 100$ array, in which cells play

\footnotetext{
${ }^{6}$ Here, the only qualifications to be noted are the randomized and clustered strategy-introduction techniques used below in order to compensate for small arrays and small computer memories.

${ }^{7}$ Although it is less clear, two-dimensional spatialization may also be playing an important role in Orbell, Runde, and Morikawa (1995, this issue).
}

neighbors in the manner outlined above. With the 121 strategies represented, such an array is very sensitive to initial conditions: much depends on which strategies are eliminated by immediate neighbors in the first few generations. Where generosity emerges, however, the common pattern seems to be evolution towards a strategy only slightly more generous than statistically imperfect TFT: usually $\langle 0.99,0.1\rangle$, though if the more generous $\langle 0.99,0.2\rangle$ survives, it can eventually supplant it. A typical evolution to $\langle 0.99,0.1\rangle$, represented by white, is shown in 12-generation intervals in Fig. 2.

It is clear that a primary factor in these first results is the limit of our array, however. Such an array inevitably imposes not only sensitivity to initial conditions, but a significantly greater 'death factor' than is present in the population proportion algorithm used in Axelrod and Hamilton, and Nowak and Sigmund. That algorithm is in fact strongly biased against extinction: although a strategy's proportion in the population may diminish at each stage, it never dies completely unless it scores a full zero against all existing strategies. None of Nowak and Sigmund's strategies has a true score of 0 against any other. In the evolution plotted in Fig. 1, the proportion of stochastically imperfect TFT falls at one point before its rise to somewhat more than half of its original representation. But the more generous $\langle 0.99,0.1\rangle$ falls to a hundredth of its original representation before recovery, $\langle 0.99,0.2\rangle$ falls to less than a millionth, and $\langle 0.99,0.3\rangle$ falls to less than a trillionth. If representation in a proportion less than that corresponding to a single individual counts as 'death', a generosity level of even $\langle 0.99,0.1\rangle$ would thus require a population in tens of thousands merely to survive, a generosity level of $\langle 0.99,0.2\rangle$ would demand a population of nearly one billion, and bare survival of a generosity level of $\langle 0.99,0.3\rangle$ would demand a population in the hundred trillions.

Within the limits of a finite array of automata of any manageable size, on the other hand, the death of a strategy can be very final very quickly. For this reason alone, although we can easily assume Nowak and Sigmund's pool of stochastic strategies and incorporate their payoff formula in 
a spatial context, a tournament of this type imposes a significantly different reproductive algorithm. Given the array limits of this first attempt at spatial modelling and the very small proportions to which more generous strategies fall in the Axelrod-Hamilton model it is perhaps not too surprising that the upper end of the Nowak and Sigmund generosity result is cut off. $\langle 0.99,0.3\rangle$ seems always to be extinguished much too early.

Here we should also mention another difference in the evolution of this first model, however. Even with a total population as small as 10000 , we have noted that convergence is standardized to a strategy slightly more generous than the statistically imperfect TFT. But the evolutionary mechanism operative in this spatialization is quite different from that in the original Nowak and Sigmund result. Within the population proportion algorithm, as indicated in Fig. 1, the pattern of the result is an early and almost total victory by stochastically imperfect TFT, followed step by step by successful invasions of more generous variations. Without the presence of stochastically imperfect TFT, Nowak and Sigmund indicate the evolution to more generous strategies cannot proceed. But that is not the characteristic evolution of the spatial model shown in Fig. 2; here it is clear that there is a direct victory by the more generous forms without the necessity of prior intervention by a statistically imperfect TFT.

\section{The evolution of greater generosity}

In two more sophisticated studies, models were designed to compensate for the effects of small arrays and small computer memories by use of a randomizing procedure, in which a limited sample of strategies compete in a given area and losers are progressively replaced by alternatives. The idea is that in a large enough array there would be areas where significant numbers of individuals from any given handful of strategies would compete, carrying the result of that competition into other areas. We simulate the larger area in bits, as it were, by progressively randomizing competing strategies into our $100 \times 100$ array. With such a procedure, the role of the death factor noted above seems properly minimized. In these more sophisticated spatial models, it is not Nowak and Sigmund's GTFT that is ultimately favored, however, but more generous strategies still.

In our second set of studies, we began with a randomized $100 \times 100$ array of just eight strategies chosen from the pool of 121. Each cell played against its neighbors as outlined above. When a strategy died - with no representatives left - a new competitor was sprinkled randomly across the field with a proportion of $1 / 8$ equal to that of the original competitors. New competitors were chosen randomly from the pool, allowing a possibility of repetition. This procedure was introduced purely for the computational reasons outlincd above, with the appearance of new strategies thought of merely as a sampling procedure across the pool of 121 strategies. Only later did we note that limitation to a fixed number of competing strategies does seem consistent with the broad outlines of the 'Theory of Island Biogeography' in biology (MacArthur and Wilson, 1967), itself sometimes offered as evidence for the Red Queen (Van Valen, 1973; Lewin, 1985).

Convergence to a particular strategy in an evolutionary ecology of this type would clearly constitute a strong argument in favor of that strategy: it must have arisen and must have maintained itself in competition with substantial distributions of large numbers of potential rivals. Given Nowak and Sigmund's work, we would not have been surprised had GTFT triumphed. But our results showed convergence quite standardly to significantly greater generosity. Convergence was almost always to a strategy in the range of $\langle 0.99,0.4\rangle$ through $\langle 0.99,0.6\rangle$, locked in equilibrium with trace elements of other strategies in such a way as to block further incursions. No strategy fully dies and thus no further strategies are introduced. The result is quite resilient. It remains (although it may take longer to develop) if we start with 16 strategies rather than eight, or introduce only $1 \%$ of an alternative strategy when one dies, or both. It remains if we deepen approximations to 0 and 1 to 0.000001 and 0.999999 , if we sharpen approximations of GTFT, or both. Fig. 3 shows a typical end-state with $\langle 0.99,0.5\rangle$ in possession, locked with trace elements of 


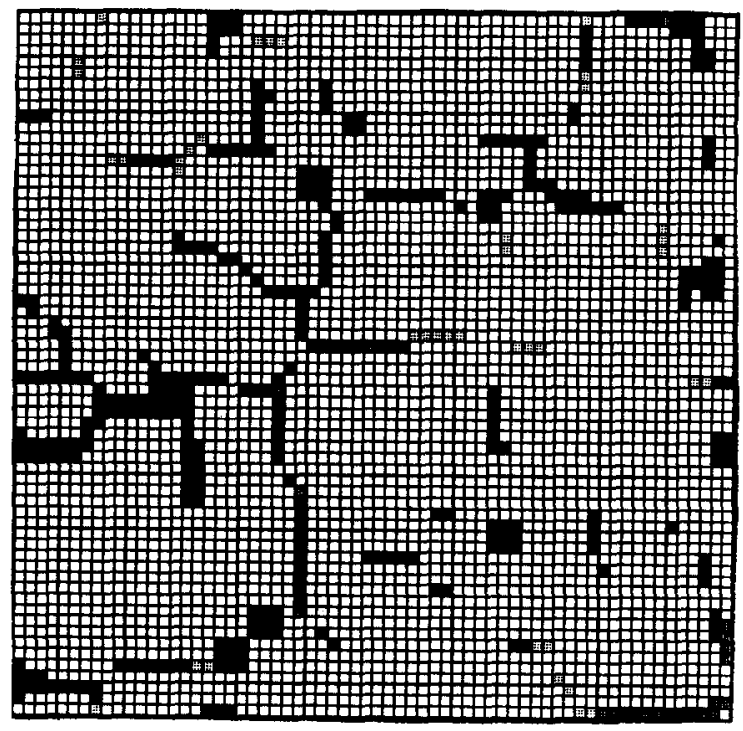

$\begin{array}{rrr}\langle .99, .50\rangle & 3696 \\ \langle .50, .01\rangle & 136 \\ \langle .60, .90\rangle & 92 \\ \langle .90, .50\rangle & 87 \\ \langle .10, .10\rangle & 38 \\ \langle .01, .30\rangle & 31 \\ \langle .60, .99\rangle & 13 \\ \langle .70, .10\rangle & 3\end{array}$

Fig. 3. A typical equilibrium dominated by greater generosity. This is generated from eight initial strategies, randomly chosen and distributed, with dead strategies replaced by randomly chosen alternatives in similar $1 / 8$ proportions.

$\langle 0.5,0.01\rangle$ and other strategies. A $64 \times 64$ display is shown for the sake of clarity.

In a third series of studies, we varied the model so as to introduce a cluster of just six units of a random strategy from each generation and to allow for a variable number of represented strategies. It was clear from the second studies that it was the chance of small clustering that was crucial to ecological dynamics, arguing for use of a clustered $0.06 \%$ rather than a randomly distributed $12.5 \%$ of the display. The use of a variable number of strategies in place of a constant eight allowed us to avoid the artificial 'locking' noted above.

Here an even clearer dominance by $\langle 0.99,0.5\rangle$ and $\langle 0.99,0.6\rangle$ was evident. In these studies, the standard result is convergence to domination by one or the other of these strategies, in clear possession of the field but with trace elements of other strategies present in equilibrium but unable to expand. Often subdominant strategies appear in the form of periodic blinkers. Fig. 4a shows $\langle 0.99,0.6\rangle$ in possession, with trace elements of $\langle 0.99,0.4\rangle,\langle 0.01,0.3\rangle$, and other strategies in equilibrium. Fig. 4b shows $\langle 0.99,0.5\rangle$ in possession with trace elements of $\langle 0.99,0.3\rangle$ and other strategies.

Within the spatial context there is one clear victory for pure GTFT: Nowak and Sigmund's $\langle 1-\epsilon, 1 / 3\rangle$ emerges quite clearly as that strategy with the highest score against itself which is impervious to spatial invasion by a single unit of any other strategy.

What the more generous experimental results emphasize, however, is that imperviousness to invasion by a single unit is not of ultimate importance in a spatial ecology of this kind. Though GTFT is impervious to invasion by a single unit of any other strategy, it does prove vulnerable to invasion by small clusters of some more generous strategies, themselves vulnerable in turn to invasion by much less generous strategies. No strategy is impervious to invasion by small clusters of all other strategies.

Within a spatial model, it becomes important to distinguish different notions of invasion, however. In particular, it proves necessary to distinguish at least invasion as: (a) growth, such that for some generation there is a succeeding generation in which there is a greater number of units of the invader; (b) sustained growth, such that for every generation there is some succeeding generation in which there is a greater number of units of the invader; (c) invasion to conquest, such that for any arbitrary area, that area is eventually occupied entirely by the invader. Fig. 5a shows two forms of self-limiting invasion: the unsustained periodic growth of a single unit of $\langle 0.01,0.01\rangle$ in a field of $\langle 0.9,0.6\rangle$, shown in one-generation intervals, and the sustained but self-limiting growth of $\langle 0.8,0.99\rangle$ in a field of $\langle 0.99,0.6\rangle$, shown for generations 1, 4, 7 and 12 . Three recurrent patterns of invasion to conquest appear in Fig. 5b, here 
a

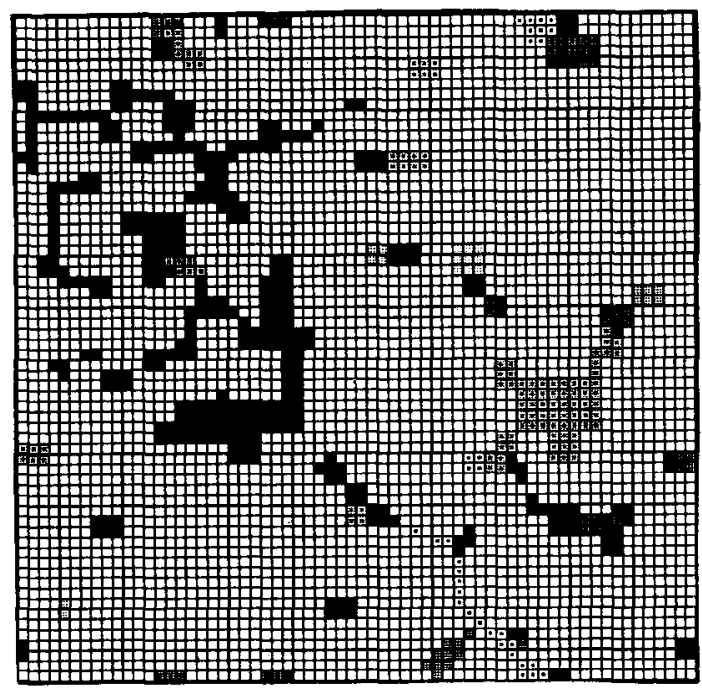

b

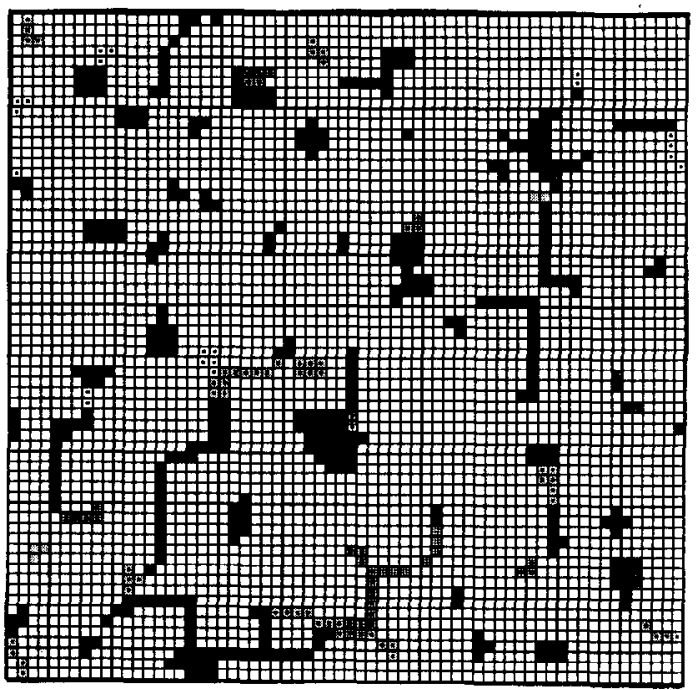

$\square \quad \begin{array}{rr}\langle .99, .60\rangle & 3507 \\ \langle .99, .40\rangle & 102 \\ \langle .01, .30\rangle & 100 \\ \langle .10, .50\rangle & 62 \\ \square .40, .20\rangle & 29 \\ \square .10, .30\rangle & 23\end{array}$

\begin{tabular}{rrr}
$\langle .99, .50\rangle$ & 3606 \\
$\langle .99, .30\rangle$ & 56 \\
$\langle .20, .60\rangle$ & 20 \\
$\langle .10, .80\rangle$ & 18 \\
$\langle .50, .70\rangle$ & 14 \\
\hline & $\langle .10, .50\rangle$ & 13
\end{tabular}

Fig. 4. Typical examples of evolution to greater generosity with a variable number of random strategies, blocks of six of a randomly chosen strategy dropped in each generation.

illustrated by $\langle 0.99,0.9\rangle$ invaded by a single unit of $\langle 0.01,0.8\rangle,\langle 0.99,0.4\rangle$ invaded by a square of nine units of $\langle 0.99,0.6\rangle$, each shown in intervals of two generations, and $\langle 0.99,0.99\rangle$ invaded by a single unit of $\langle 0.5,0.6\rangle$, shown for generations 1 , 4, 8 and 12. A GTFT of $\langle 0.9999999,0.3333333\rangle$ is invadable to conquest by clusters as small as 4 units of, for example, $\langle 0.9999999,0.5\rangle$. It is also invadable, though not to conquest, by for example $\langle 0.9999999,0.9999999\rangle$.

The complex dynamics of this second invasion, as it happens, exhibits in a stochastic and somewhat less artificial context many of the features of Nowak and May's (1992, 1993) work on spatial chaos and fractals in evolutionary games. The growth of a pattern of 16 units of AllC in a field of GTFT with this amount of stochastic imperfection is shown at intervals of six generations in Fig. 6 , with coding in grey to indicate dynamics: black indicates a cell of the invading strategy which has not changed in the last round; white, a cell of the invaded strategy which has not changed; light grey, a cell which has been invaded in the last round; and dark grey, a cell that has reverted to the invaded strategy ${ }^{8}$.

GTFT, we've noted, though invulnerable to invasion by a single unit of alternative strategies, is vulnerable to invasion by small clusters of some more generous strategies. In this way, it resembles pure non-stochastic AllD which, though not

\footnotetext{
${ }^{8}$ The intricacy of this illustration is due to the precarious balance between strategies at issue, and its symmetry to the fact that all cells of the array are updated simultaneously. Use of an asynchronous updating - in which, say, a random $50 \%$ or $25 \%$ of the display updates at each step-can therefore be expected to give a much less dramatic result (Huberman and Glance, 1993).
} 
a
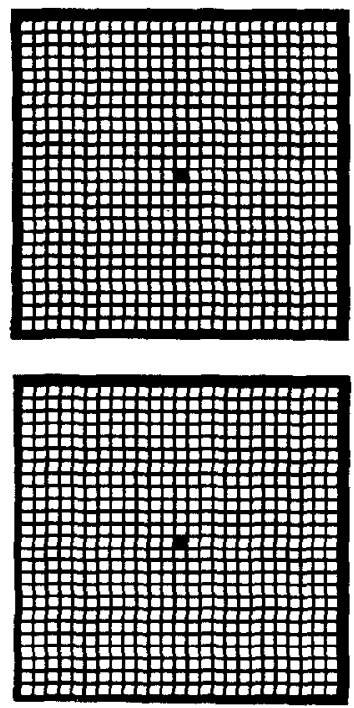

b
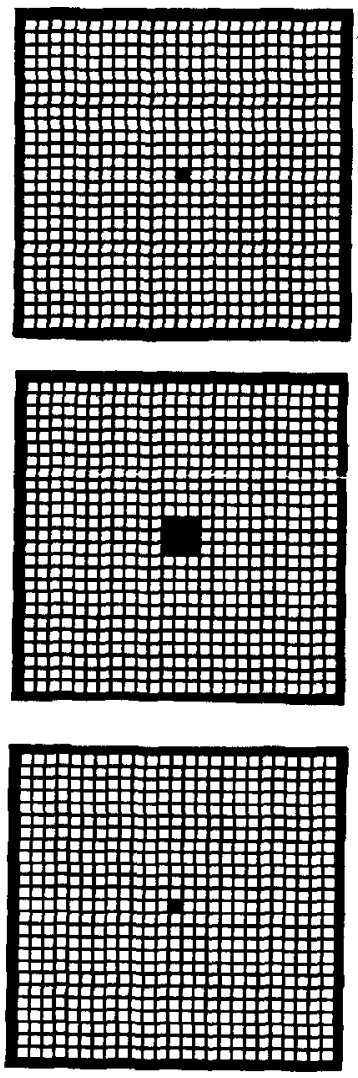
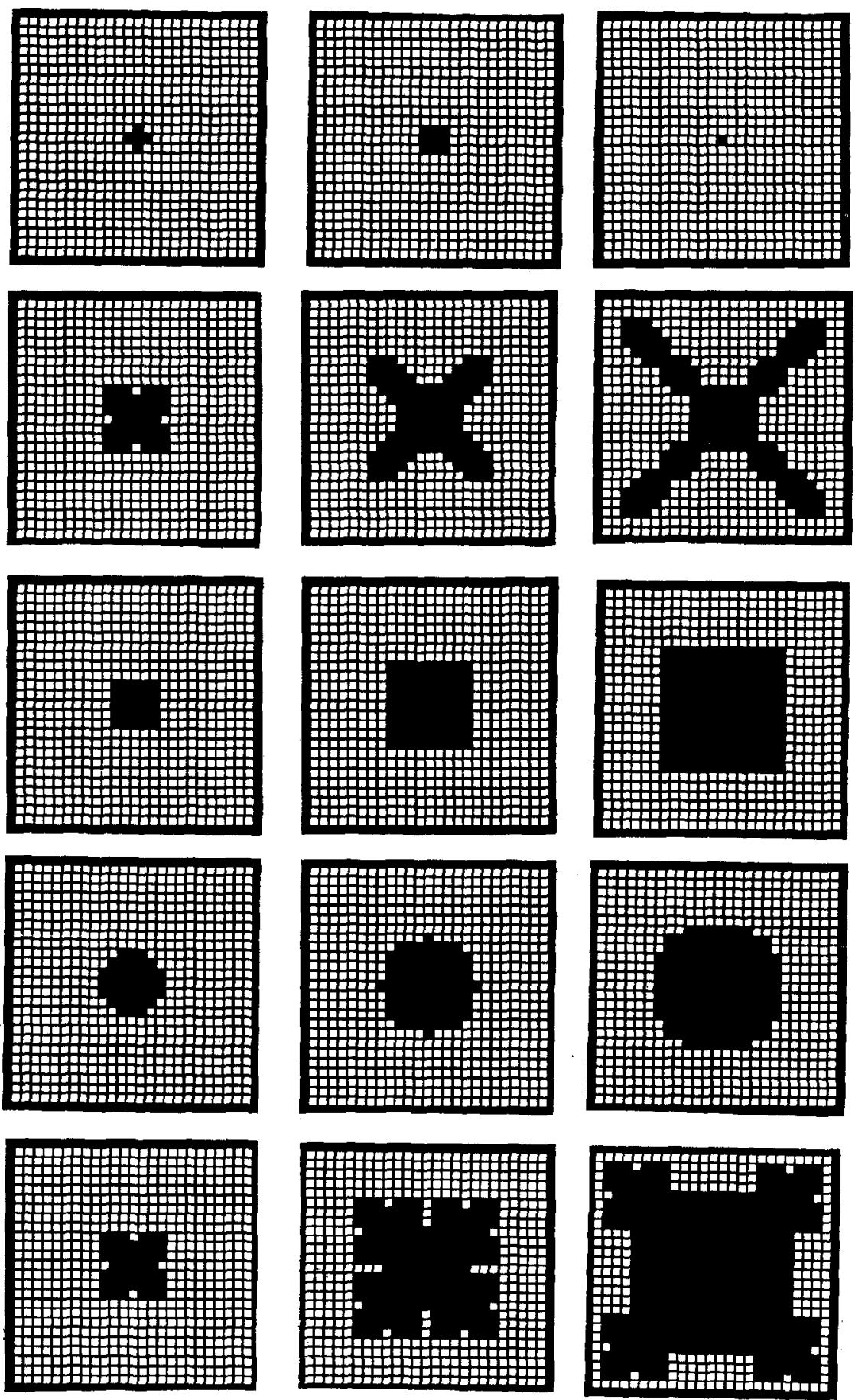

Fig. 5. (a) Two common forms of self-limiting expansion; (b) three patterns of evolution to conquest. 

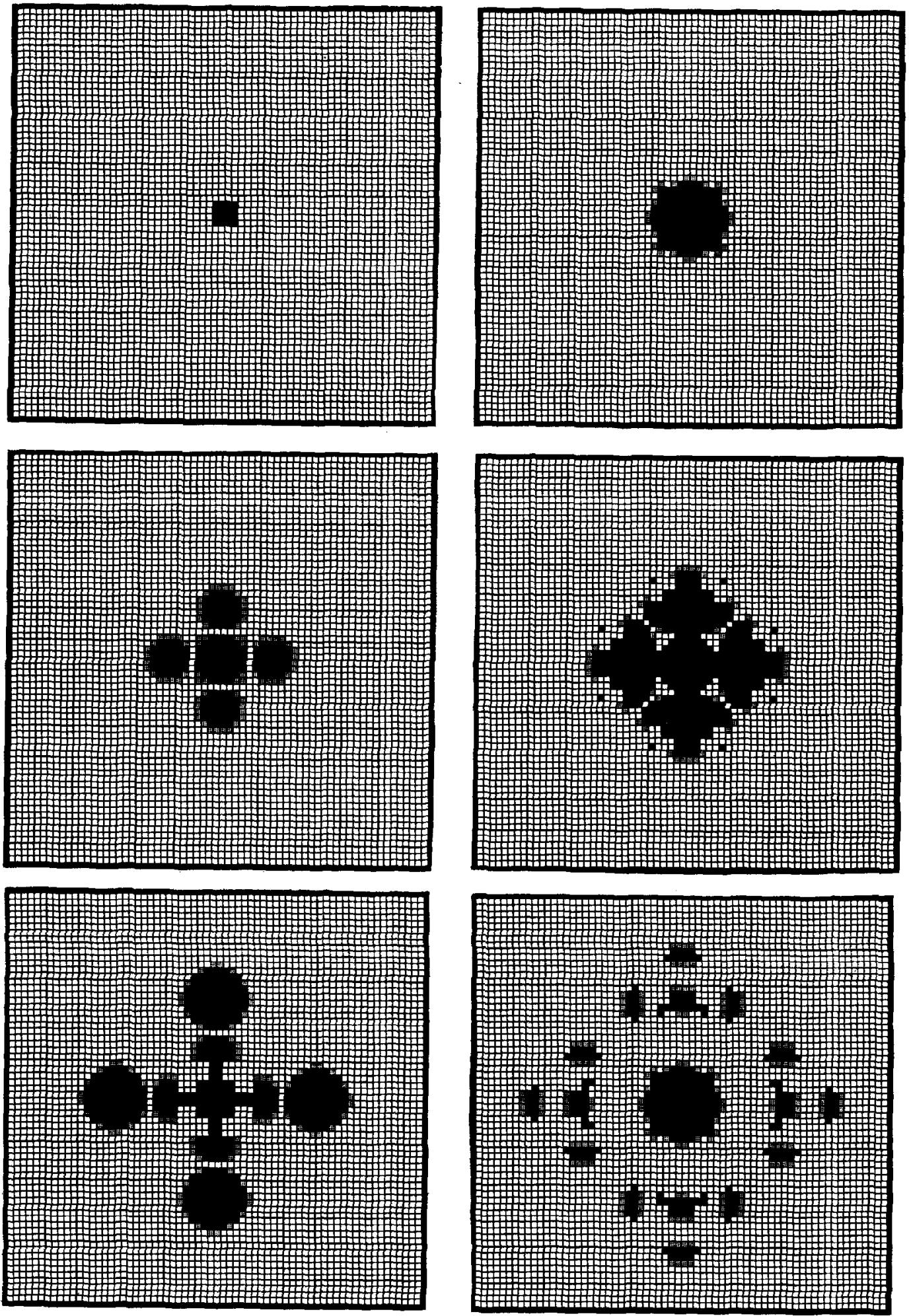

Fig. 6. KA field of $\langle 0.9999999,0.3333333\rangle$ invaded by a 16 -square block of $\langle 0.9999999,0.9999999\rangle$, shown at intervals of six generations. 

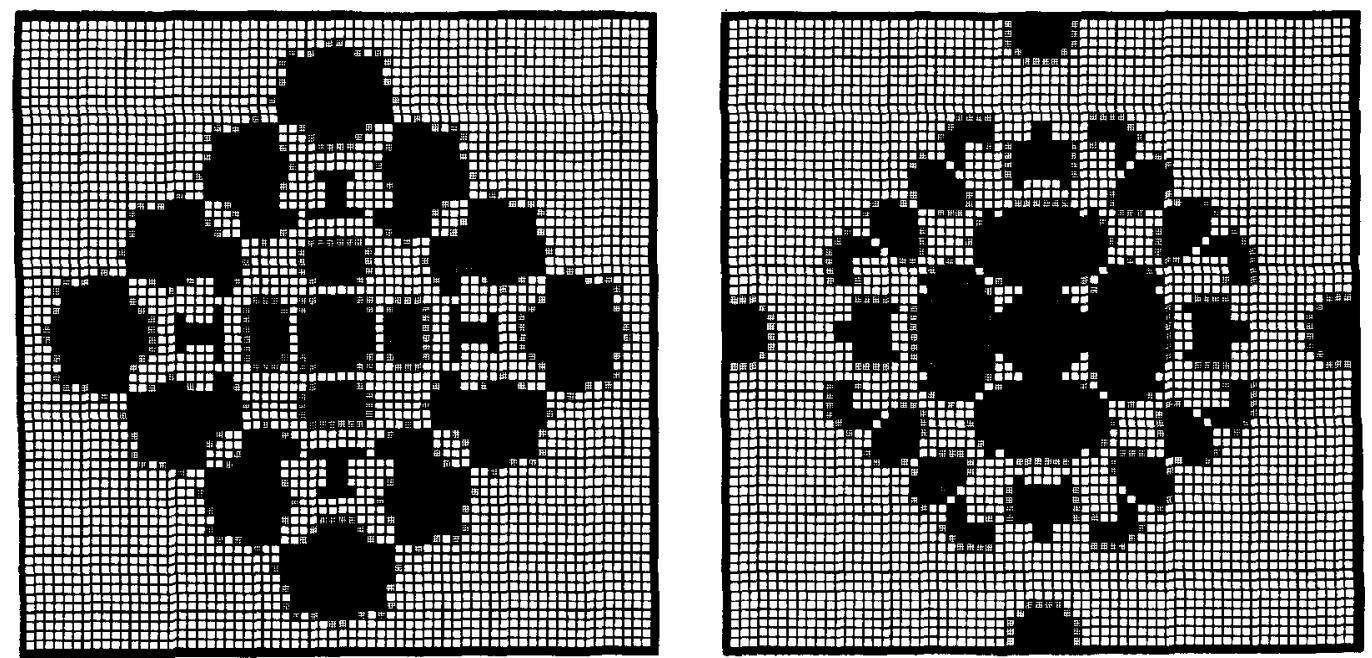

inuading strategy, unchanged last round

invaded strategy, unchanged last round

changed from invaded strategy to invading strategy last round

changed from invading strategy to invaded strategy last round

Fig. 6. continued

invadable by a single unit of pure TFT, is invadable by small clusters of TFT. Strategies more generous than GTFT, on the other hand, up through $\langle 0.9999999,0.6666666\rangle$, are clearly invadable by other strategies but seem invadable to conquest by no others in at least standard patterns from small clusters?.

In our computerized surveys the strategic region $\langle 1-\epsilon, 2 / 3\rangle$, which we term 'bending over backwards', seems to emerge as that of the experimentally optimal strategy, in the sense of that strategy with the highest score against itself impervious to invasion to conquest from a small cluster of any other strategy. In the hierarchical rule formulation suggested by Crowley (1995, this issue), 'bending over backwards' can be character-

\footnotetext{
${ }^{9}$ Herc, rectangular blocks of 2 and 6 , crosses of 4 , and square blocks of 4,9 , and 16 were used as initial invading clusters, with surveys programmed to indicate whether invasion had proceeded to a particular border in a chosen number of generations. As for invasion patterns more generally, however, more analytic work remains to be done.
}

ized as:

$/: \mathrm{C}$

$/ \mathrm{D}: \mathrm{D}$

$/ \mathrm{D}: \mathrm{C}$

$/ \mathrm{D}: \mathrm{C}$

The work above builds explicitly on Nowak and Sigmund (1992), and is similarly limited to onedeep or reactive strategies, which consider only the previous move of the opponent. We leave further study of spatialization for games which consider previous moves of both players, such as Pavlov (Nowak and Sigmund, 1993), and for optional games (Kitcher, 1993; Batali and Kitcher 1994; Orbell, Runde and Morikawa, 1995, this issue; Ashlock, Smucker, Stanley and Tesfatsion, this issue) to others or to another paper. Variations employing asynchronous updating (Huberman and Glance, 1993) are also left for further work, though on an experimental basis we have tried a limited form of asynchronous updating for results throughout by computing only a random 
$50 \%$ or $25 \%$ of arrays on each generation ${ }^{10}$. As might be expected, precise patterns of propagation dependent on the particular configuration of a group, such as those in Figs. 5 and 6, prove vulnerable to asynchronous updating. Within the experimental limits noted, however, results regarding the greater success of generous strategies in a spatial environment seem to remain. It may also be that the stochastic character of strategies at issue diminishes differential effects of synchronous and asynchronous treatment (see Golze, 1978).

A diskette with major programs in Trubasic is available on request.

\section{Conclusion}

In the Axelrod models for a pure and non-stochastic Prisoner's Dilemma, it is TFT that proves the general winner. Within a stochastic model designed to capture features of a world of error and imperfect communication, the greater generosity of GTFT pays off. With just one further measure of realism - a two-dimensional spatialization of the stochastic model - it is a strategy of 'bending over backwards' which seems to prove victorious, exhibiting twice the generosity of GTFT.

\section{Acknowledgements}

I am grateful to Gary Mar and Paul St. Denis for sharing their seminal work on cellular automata and the Prisoner's Dilemma in the non-stochastic case, to Martin Nowak for encouragement, and to Paul St. Denis, Tobias Müller, and David Gill for programming assistance. An earlier and less complete report of these results appears as Grim 1994.

\footnotetext{
${ }^{10}$ Huberman and Glance (1993) seem to insist on updating at most a single cell each generation, but a fixed choice of one cell seems no less artificial than simultaneous updating of all cells. Some form of weighted randomization would seem far more preferable, though what form remains an open question and might even depend on the type of species we are interested in modelling.
}

\section{References}

Ashluck, D., Smucker, M., Stanley, E. and Tesfatsion, L., 1996, Preferential partner selection in an evolutionary study of Prisoner's Dilemma. BioSystems, 37, 99-125.

Axelrod, R. 1980a, Effective choice in the Prisoner's Dilemma. J. Conf. Resolut. 24, 3-25.

Axelrod, R., 1980b, More effective choice in the Prisoner's Dilemma. J. Conf. Resolut. 24, 379-403.

Axelrod, R., 1984, The evolution of cooperation (Basic Books, New York).

Axelrod, R., 1985, The emergence of cooperation among egoists, in: Paradoxes of Rationality and Cooperation, $T$. Campbell and L. Sowden (eds.) (Univ. of British Columbia Press, Vancouver).

Axelrod, R. and Hamilton, W., 1981, The evolution of cooperation. Science 211, 1390-1396.

Batali, J. and Kitcher, P., 1995, Evolution of altruism in optional and compulsory games. J. Theor. Biol., in press.

Crowley, P., 1996, Evolving cooperation: strategies as hierarchies of rules. BioSystems, 37, 67-80.

Crowley, P., Provencher, L. Sloane, S., Dugatkin, L., Spohn, B., Rogeres, L. and Alfieri, M., 1996, Evolving cooperation: the role of individual recognition. BioSystems, 37, 49-66.

Demongeot, J., Golés, E. and Tchuente, M., (eds.) 1985, Dynamical systems and cellular automata (Academic Press, New York).

Dugatkin, L. and Mesterto-Gibbons, M., 1996, Cooperation among unrelated individuals: reciprocal altruism, byproduct mutualism and group selection in fishes. BioSystems, 37, 19-30.

Golze, U., 1978, (A-)Synchronous (non-)deterministic cell spaces simulating each other. J. Comput. Sys. Sci. 17, 176-193.

Grim, P., 1995, Greater generosity in the spatialized Prisoner's Dilemma. J. Theo. Bio. 173, 353-359.

Gutowitz, H., (ed.) 1990, Cellular automata: Theory and experiment (North-Holland, New York).

Huberman, B. and Glance, N., 1993, Evolutionary games and computer simulations. Proc. Natl. Acad. Sci. USA 90, 7716-7718.

Kitcher, P., 1993, The evolution of human altruism. J. Philos. 90, 497-516

Lewin, R., 1985, Red queen runs into trouble? Science 227 , 399-400.

Macarthur, R. and Wilson, E., 1967, The theory of island biogeography (Princeton University Press, Princeton, NJ)

Mar, G. and St. Denis, P., 1994, Chaos in coopcration: continuous-valued Prisoner's Dilemmas in infinite-valued logic. Int. J. Bifurcation and Chaos 4, 943-958.

Maynard Smith, J., 1982, Evolution and the theory of games (Cambridge Univ. Press, Cambridge, MA).

Maynard Smith., J., 1986, Evolutionary game theory, in: Evolution, Games, and Learning: Models for Adaptation in Machines and Nature. Proceedings of the Fifth Annual International Conference of the Center for Non-linear 
Studies, Los Alamos, 1985, D. Farmer, A. Lapedes, N. Packard and B. Wendroff (eds.) (North-Holland, Amsterdam)

Molander, P., 1985, The optimal level of generosity in a selfish, uncertain environment. J. Conf. Resolut. 29, 611-618.

Moon, T., Frost, R. and Stirling, W., 1996, An epistemic utility approach to coordination in the Prisoner's Dilemma. BioSystems, 37, 167-176.

Nowak, M., 1990a, Stochastic strategies in the prisoner's dilemma. Theor. Popul. Biol. 38, 93-112.

Nowak, M., 1990b, An evolutionarily stable strategy may be inaccessible. J. Theor. Biol. 142, 237-241.

Nowak, M. and May, R., 1992, Evolutionary games and spatial chaos. Nature 359, 826-829.

Nowak, M. and May, R., 1993, The spatial dimensions of evolution. Int. J. Bifurcation Chaos 3, 35-78.

Nowak, M. and Sigmund, K., 1989a, Game-dynamical aspects of the Prisoner's Dilemma. Appl. Math. Computat. 30, 191-213.

Nowak, M. and Sigmund, K., 1989b, Oscillations in the evolution of reciprocity. J. Theor. Biol. 137, 21-26.

Nowak, M. and Sigmund, K., 1992, Tit for tat in heterogeneous populations. Nature $355,250-252$.
Nowak, M. and Sigmund, K., 1993, A strategy of win-stay, lose-shift that outperforms tit-for-tat in the prisoner's dilemma game. Nature 364, 56-58.

Orbell, J., Runde, A., Morikawa, T., 1996, The robustness of cognitively simple judgement in ecologies of Prisoner's Dilemma games. BioSystems, 37, 81-97.

Oliphant, M., 1994, Evolving cooperation in the non-iterated Prisoner's Dilemma: The importance of spatial organization, in: Artificial Life IV (MIT Press, Cambridge, MA), pp. 349-352.

Oliphant, M., 1996, The dilemma of Saussurean communication. BioSystems, 37, 31-38.

Sandholm, T. and Crites, R., 1995, Multiagent reinforcement learning in the Iterated Prisoner's Dilemma. BioSystems, $37,147-166$.

Skyrms, B., 1992, Chaos in game dynamics. J. Logic, Lang. Inf. 1, 111-130.

Van Valen, L., 1973, A new evolutionary law. Evolut. Theor. 1, 1-30.

Wolfram, S., 1984, Cellular automata as models of complexity. Nature 311, 419-424.

Wolfram, S., 1986, Theory and applications of cellular automata (World Scientific, Philadelphia). 